\title{
SIFT based Dorsal Vein Recognition System for Cashless Treatment through Medical Insurance
}

\author{
Rajendra Kumar, Ram Chandra Singh, Ashok Kumar Sahoo
}

\begin{abstract}
The time has come not to carry any physical personal identity. The person's identity will be known by his/her biometric features for any purpose exclusively when the person is unconscious and not carrying any identity proof. Vein recognition system is leading biometric trait in terms of flexibility and security. The fake recognition of veins is almost impossible as the feature points lie underneath the skin and cannot be read without the knowledge of a person (if not unconscious). Most important thing about it is that vein recognitionsystem works only in living persons. In this paper, a recognition system is proposed that works on dorsal vein to claim cashless treatment in a hospital which is on panel of medical insurance company. For acquisition of vein image, a NIR camera VF620 of $850 \mathrm{~nm}$ wavelength is used. The proposed model is applied on 1000 samples of dorsal vein patterns of 250 persons. The overall performance of the system was observed to be $97.95 \%$ with EER (Equal Error Rate) $0.0435 \%$.
\end{abstract}

Index Terms: Veins Pattern Recognition, Biometric Identification, Dorsal Vein Biometric, SIFT, Pattern Recognition.

\section{INTRODUCTION}

For every identification, security and convenience are the prime objectives. If the identity features in a biometric identification are inside the body and cannot be extracted without the consent of a person then it becomes more secure. Vein pattern recognition and identification give such kind of flexibility [1]. It has already been proved that vein patterns are unique even in twins [2] and therefore difficult to forge. Additionally, it has no effect in matching whether there is case of skin discoloration, light conditions, minor distance variation from camera to dorsal, etc. [3]. The contactless feature makes it more better than fingerprint recognition.

Several challenges have been observed in different biometric recognitions, like scaling segmentation, quality and other physical conditions in latent fingerprints; Quality of scanner, co-operation by user with recognition system, etc. To overcome the problem of scaling, the SIFT (scale invariantfeature transform) algorithm have shown very good results.

The identification of a person was started with ICard, Password, PIN, etc. But these were not reliable with time as these can be stolen, unknowingly disclosed and forgot. A K Jain et al.[4] have shown that as the technology progressed, the physical characteristics/biological features (like fingerprint, palm print, iris, face, etc.) came into effect and commercially applied for authentication. With the time it is notices that the unauthorized duplication of fingerprint and

Revised Manuscript Received on July 20, 2019

Rajendra Kumar, Research Scholar, Department of Computer Science and Engineering, School of Engineering and Technology, Sharda University, Greater Noida, India,

Ram Chandra Singh, Department of Physics, School of Basic Sciences and Research, Sharda University, Greater Noida, India.

Ashok Kumar Sahoo, Department of Computer Science and Engineering, Graphic Era Hill University, Dehradun, India. palm prints spoofed the authentication system [4] came in knowledge.To avoid the unauthorized duplication of fingerprints and palm prints, it is highly desirable to use the person's biological features that cannot be reproduced without the consent of the person [5]. This led the researchers to use veins pattern (palm, finger, etc.) as one of the biometric traits [6]. Bango Chao Liu et al. used LBP (Local Binary Pattern) for vein recognition [6]. Sen Lin et al. focused on finding ROI (region of interest) to get better feature point extraction for high matching [7]. To improve the security,Parthsarthiet.al. [8] used dual authentication and Jaekwon Lee et al. [9] used liveliness of the user to detect spoofing. Montiel et.al.used segmentation for better recognition [2].

Several biometric traits have been developed so far like fingerprint, iris, face, etc. [10]. Maleika et al. used palm vein patterns as biometric recognition as it has more area to have sufficient feature points [11]. Every trait has its advantages and disadvantages and based on that they are implemented commercially. In India, fingerprints are being used in AADHAR card [12]. Iris is being used at airports. In recent years many cases of fingerprint imitations have been reported and therefore it cannot be treated as a most secure biometric trait. A person's fake fingerprint can be misused for different purposes. The fake vein patterns cannot be developed by any means as it is captured by NIR camera and person's physical appearance is must for same.

Hardika et al. proposed multi-feature fusion finger vein recognition using SIFT and LEBP (local extensive binary pattern). This method overcame the problem of image degradation due to image operations like scaling, rotation and translation. It has been observed that variation in illumination creates problem in feature extraction by SIFT[13]. To overcome this problem Wang et al. [14] used LEBP as it hasfeature to resist variation in illumination conditions. They also proposed SIFT based vein recognition model focusing on contrast enhancement $(\mathrm{CE})$.

Hans Verghese Mathews et al. presented flaws in the UIDAI Process by mentioning major challenge in unauthorized uses [15].Naidile et al. [16] proposed a dorsal hand vein recognition system. Simple correlation technique was used by them for matching purpose. The system presented in [16] produced positive detection rate of $75 \%$ and negative detection rate was $25 \%$.

Wang et al.[14]discussed that scale-invariant feature transform (SIFT) is being investigated more and more to realize a less-constrained hand vein recognition system. Contrast enhancement (CE), compensating for deficient dynamic range aspects, is a must for SIFT based framework to improve the performance. 
However, evidence of negative influence on SIFT matching brought by $\mathrm{CE}$ is analyzed by their experiments [17]. Sen Lin et al. [18] focused on methods of extracting region of interest (ROI) for palm vein recognition

Mulyono et al.[19] used finger vein grabber device SMT780 (Marubeni Corporation's product) with wavelength $780 \mathrm{~nm}$ for image capturing. Raghvendra et al. [12] used a sensor based on a near infrared illumination that can emit light in a spectrum of $940 \mathrm{~nm}$ that in turn is used to illuminate the dorsal hand region.Kumar et al. [20] applied triangulation and shape feature on personal database of dorsal vein patterns to produce EER $1.14 \%$. Antonio et al. [21] used minutiae extraction algorithm on Singapore database of dorsal vein patterns to produce EER 1.63\%. Yuksel et al. [22] used geometric and appearance based fusion on Bosphorus database and produced EER $1.98 \%$.

The motivation behind this study is that the vein patterns are unique even in twins. This technology works only on live persons as hemoglobin in veins absorbs the infrared light and the veins appear black to be captured for identity. Since,vein patterns are underneath the skin therefore capturing of vein pattern is difficult without consent of user. Its contactless feature makes it durable and hygienic. Last but not the least its commercialization is very limited.

The paper is organized as follows.Section II presents the model of the proposed system. The implementation of the proposed system is discussed in Section III. Section IV presents the results and Discussion and finally, section $\mathrm{V}$ concludes the outcome of this work along with the future scope.

\section{THE PROPOSED MODEL}

The proposed system is aimed to provide the cashless treatment facility in the hospitals which are on panel of insurance company. The system contains three major sections: insurance company, hospital and policy holder.

Insurance company - The insurance company insures a customer by taking documentdetails like name, date of birth, phone number, address, family details and captures dorsal vein pattern (by NIR Camera) of left and right side for identification. The medical insurance company issues insurance policy to customer that contains all the details of customer and the risk covers along with the list of hospital on its panel.

Hospital - The hospitalmaintains list of insurance companies to which hospitals are associated. When a new medical insurance is issued to a customer, the details of customer along with the vein patterns (in encrypted form) is accessed by the hospital from insurance company server. Some such systems are already in use, but in different way like, Bethanny et al. [23] used finger vein data of all patients to keep their record for further biometric identification.

Policy Holder - There are two ways in which a policy holder can be treated: (i) Planned cashless treatment (as some disease is diagnosed) and (ii) Causality (as insurance policy holder meets with some causality).

In planned cashless treatment, the insured user contacts to hospital for treatment of decease as diagnosed. The hospital scans the dorsal veins of patient by NIR camera attached to the system. The system instantly identifies the customer and shows the details of patient and fixes an appointment for treatment. The details of patient, treatment duration, treatment cost and other status are updated at insurance company server. The DFD (data flow diagram) in Figure 1 shows how this system associates a policy holder (insured user) with insurance company and hospitals on its panel.

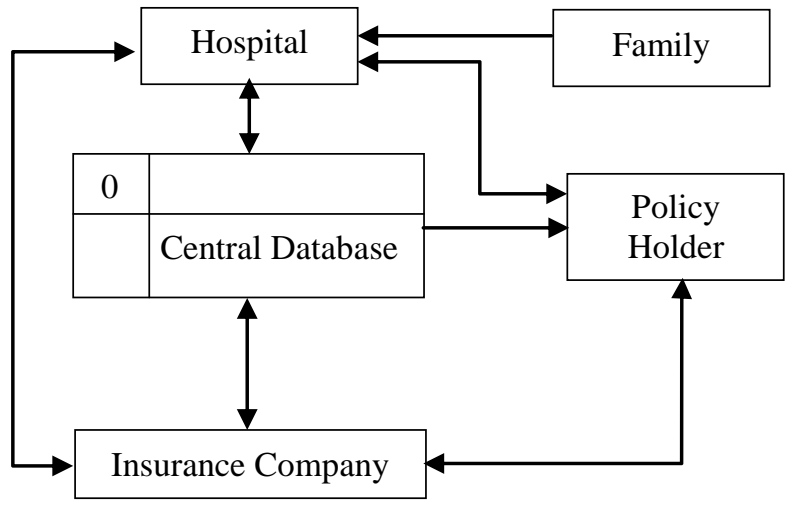

Fig. 1. 0-level DFD of the system

In case of causalities, if an unconscious person is found without any ID, is supposed to be taken to hospital. May be person met with some crime and person's ids were destroyed or stolen and the person is in unconscious condition. This case is represented by data flow diagram shown in Figure 2.

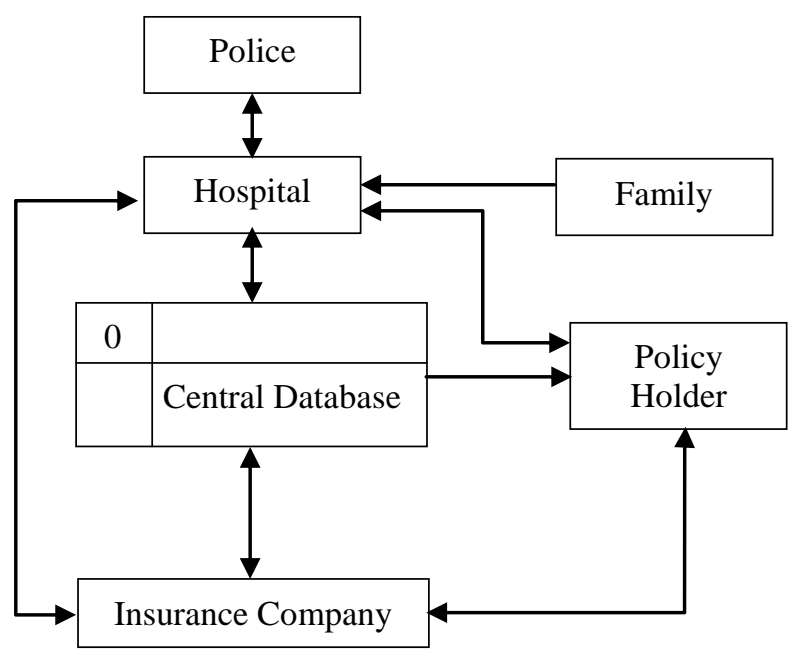

Fig. 2. 0-level DFD of the system

The person under causality is supposed to be sent to nearest hospital by someone. The hospital person scans the patient's dorsal and system tells: whether the person is under medical insurance; what risks are covered and upto what amount; person is eligible to which hospitals for cashless treatment. The system sends update on every scan of dorsal vein to the insurance company for security purpose so that no hospital able to do any fraud. Instantly, all the details of the person will be displayed on the system if he/she is medically insured already. If the hospital is not on panel of insurance company from which the person is insured then the person will be sent to nearest hospital on panel for treatment and the family of that person will be informed by the system through automatic email and SMS. If no record of patient is found, then person will be referred to government hospital and case will be informed to police for further action.

Figure 3 shows the flow chart representation of Figure 2.

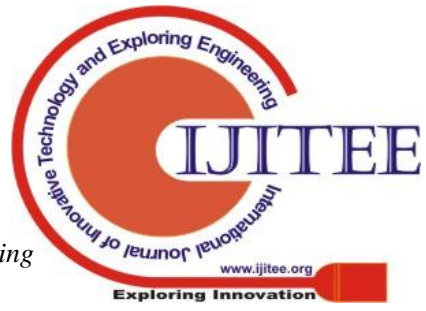


When the unconscious person is taken into hospital, the hospital first identifies the person's identity based on dorsal vein pattern with the central database of dorsal vein patterns of insured persons. If the person is medically insured then cashless treatment will be started and simultaneously the system will send message/email alerts to insurance company, insurance agent and person's family. If the person is not identified then hospital will inform to police for identification and treatment.

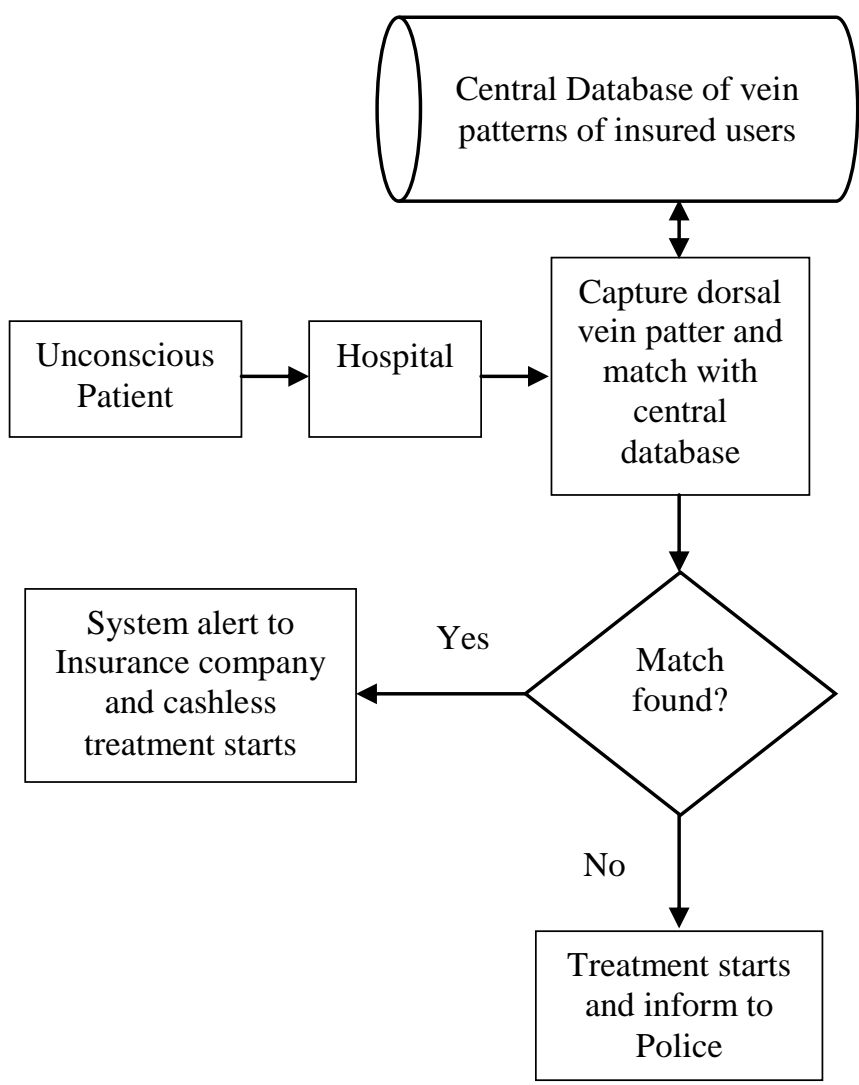

Fig. 3. Verification of unconscious patient in Hospital

Based on details and capturing of dorsal vein pattern of insured person, the central database is updated. Now the policy holder (insured person) can create his/her account to know the details of insurance and claim when required. The hospital modules can access the updated records as per insertion/deletion of records in central database. The record of a user is disabled if the insurance is not renewed within the deadline. Such users can be identified but they are not entitled for cashless treatment.

Policy Holder - As a new person is insured by an insurance company, his/her details are added to the system. Same updated details will now be accessible to hospitals. The insured person creates his/her account in the system by entering the policy number and registered mobile number/email shared with the insurance company. The system sends an OTP (one-time password) to registered mobile number/email. After entering the OTP, the system displays all the information of user which are shared with the insurance company. Now the user will be asked to enter desired password for future access. The policy number is used as the default user identity. The flow chart account creation at user end and hospital end (newly established) is shown in Figure 4.
Hospital-If a new hospital is open and wish to associate with some insurance companies, the hospital can create an account in the system. After physical verification, the hospital is assigned a hospital code and shared with policy holder through updated system.

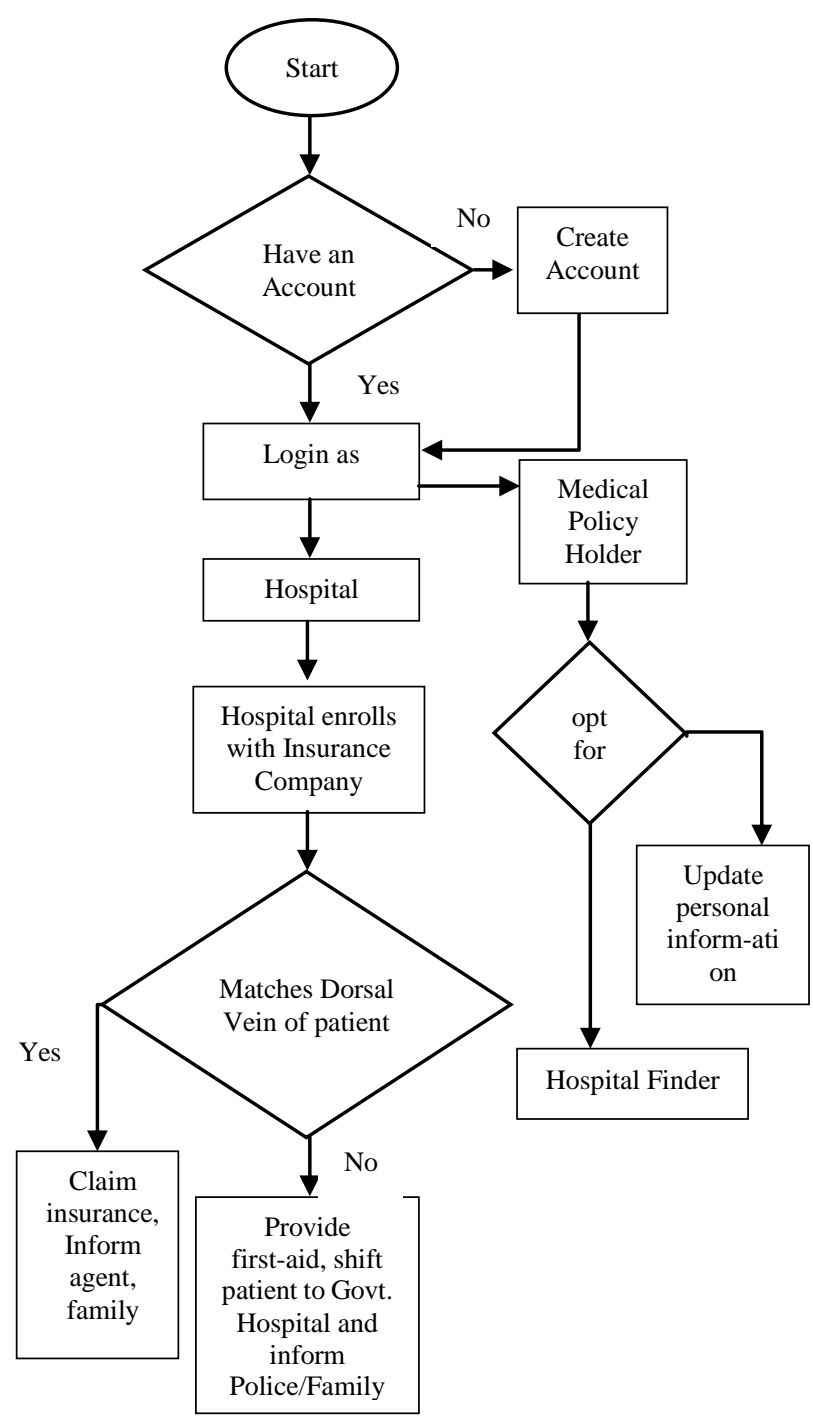

Fig. 4. Flow chart for user/hospital account creation

The data flow diagram of the entire system is presented in Figure 5, below. 


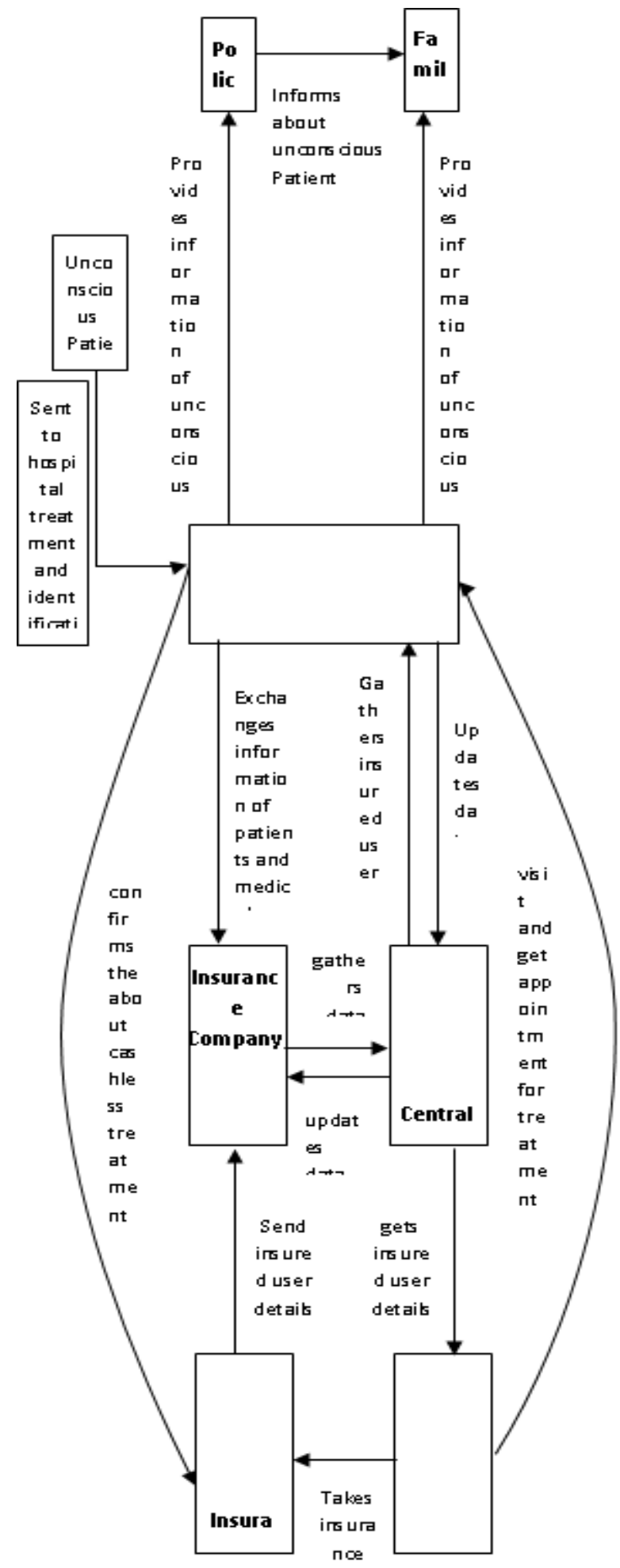

Fig. 5. DFD of the proposed system

\section{IMPLEMENTATION}

\section{A. Software Specification}

Python 3.7.3 with OpenCv package is used as software support on Win 10 platform.

\section{B. Hardware Specification}

The system needs around $5 \mathrm{Mb}$ space for storage of program code and $215 \mathrm{~KB}$ for four images ( 2 images of left dorsal and 2 images of right dorsal) per person. The system executed on Intel(R) Core ${ }^{\mathrm{TM}}$ i5-7200U CPU with $2.5 \mathrm{GHz}$ and 8GB RAM.

\section{Data Acquisition}

The hardware used for capturing the vein patterns is NIR camera (VF620) of wavelength lying between 700 to 1000 $\mathrm{nm}$. When infrared rays of this range passes through human tissues, the hemoglobin in blood absorbs the infrared radiation. As a result, the vein patterns can easily be captured.It is contactless function giving the helpful advantage (hygienic) over fingerprint and palm print [24, 25].

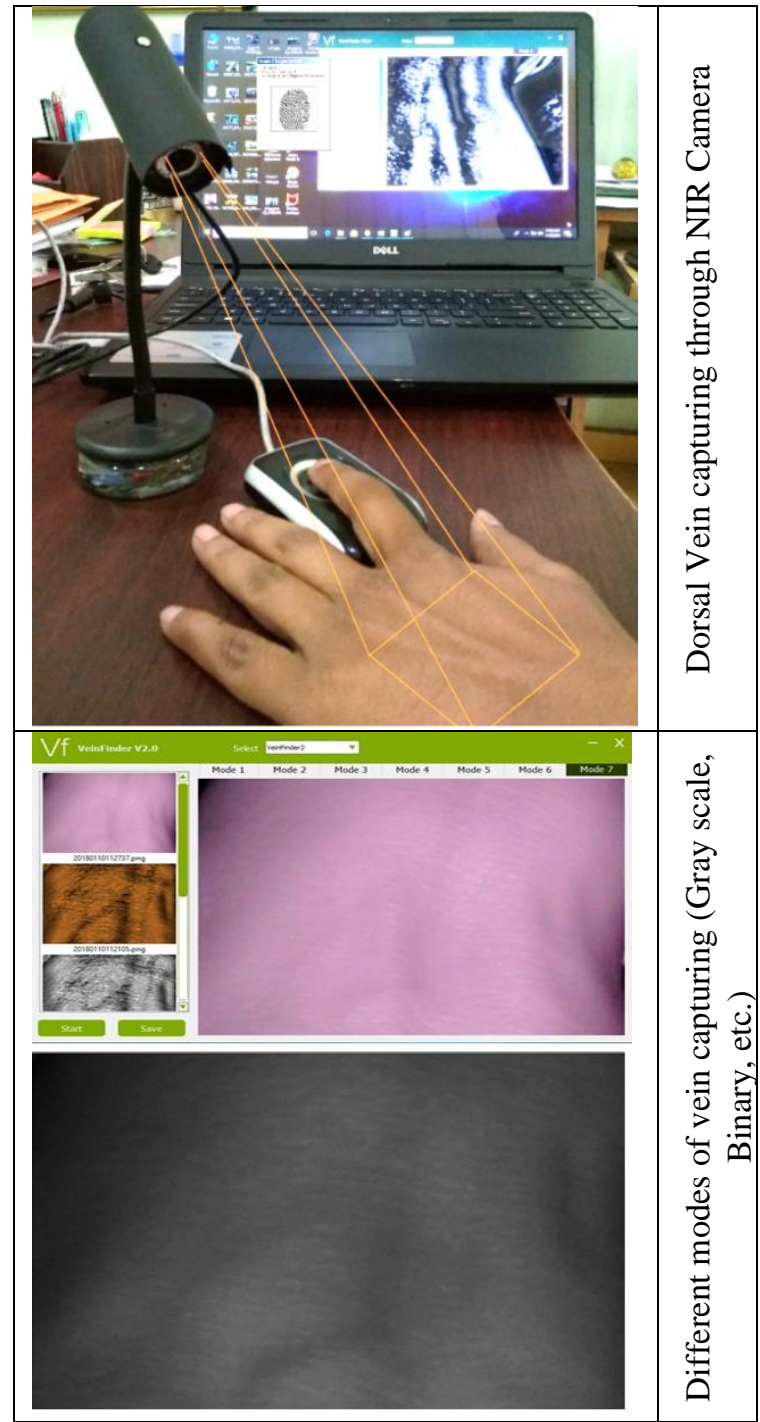

Fig. 6. Vein pattern capturing during enrollment

A near infrared camera (NIR) is used to capture the veins pattern. When the finger/palm is illuminated with infrared radiation, the veins appear black and same are recorded as identity attributes in a biometric system and further can be used for comparison during authentication [26]-[28]. The process of data acquisition involves use of appropriate software and hardware.

\section{Image Specification}

Images of size $320 \times 240$ in .png format are used in experiments. The dorsal vein patterns of 250 persons including 80 male adults, 80 female adults and 90 kids of age 05 years to 12 years are captured. 


\section{E. Object Collection}

The dorsal vein captured during enrollment is shown in Figure 6. Sample images of male adults, female adults and kids are used in the experiments. Vein pattern sample of male user is given in Figure 7. The information like name, date of birth, contact number, address and date of capturing.

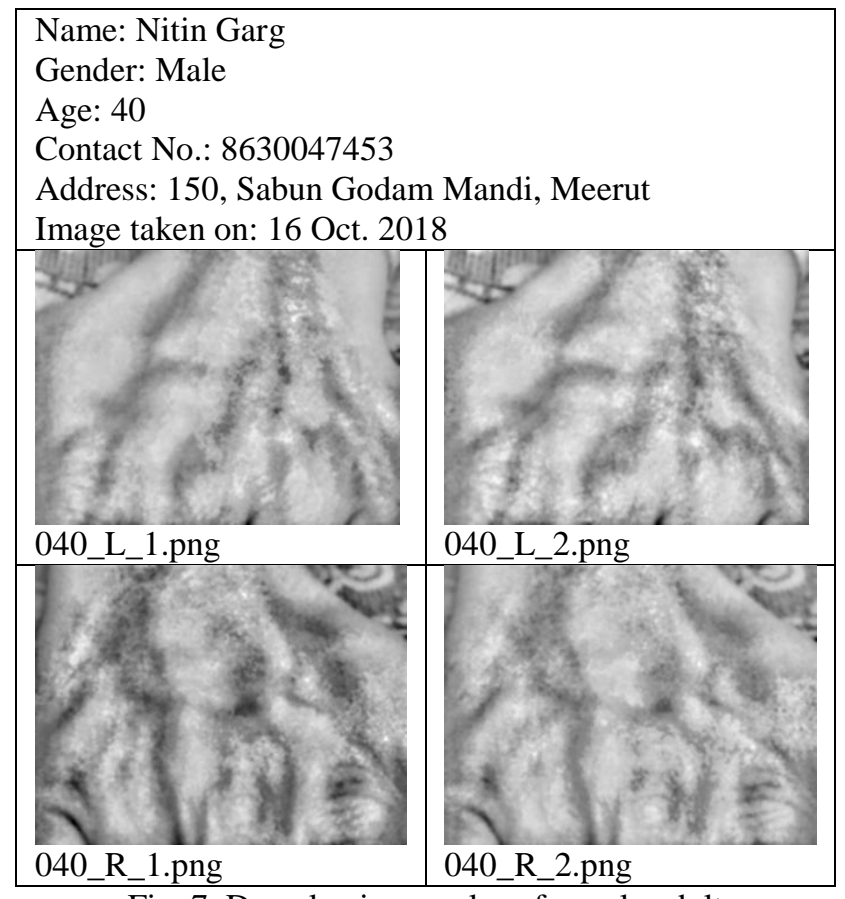

Fig. 7. Dorsal vein samples of a male adult

Vein pattern Sample of Female user is given in figure 8.

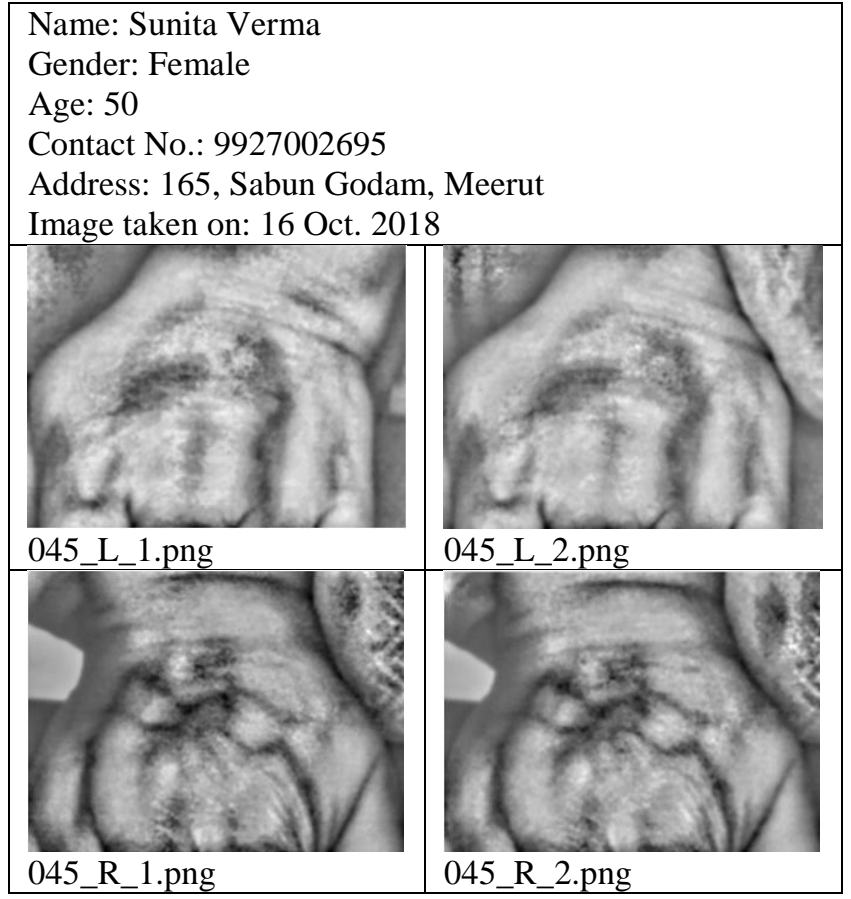

Fig. 8. Dorsal vein samples of a female adult

Vein pattern Sample of kid user is given in Figure 9.

Child Name: Disha Aggarwal

Age: 9 yrs.

Gender: Female

Father Name: Mr. Pukar Aggarwal

Contact Number: 8958913120

Address: 168, Sabun Godam, Meerut

Image taken on: 16 Oct. 2018

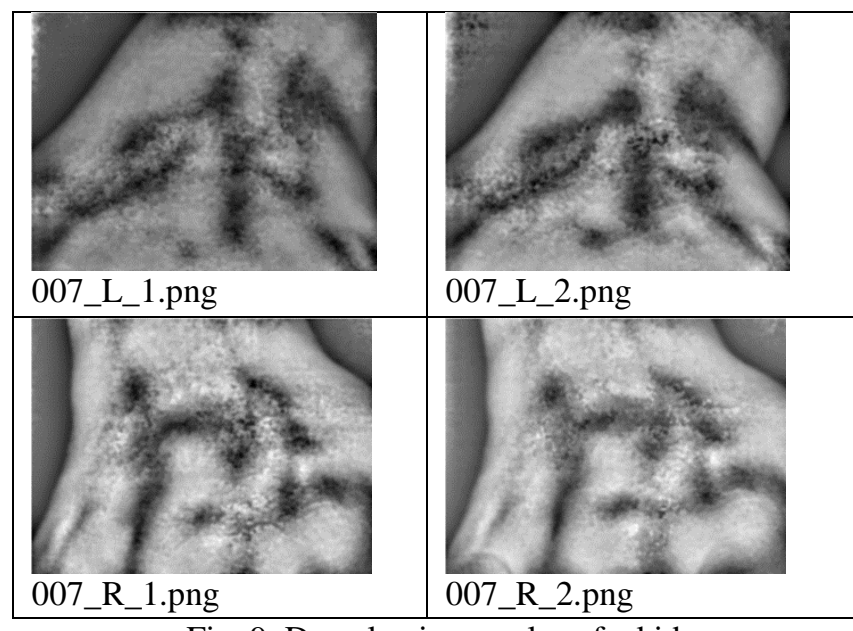

Fig. 9. Dorsal vein samples of a kid

\section{F. Persons' Identification}

Dorsal Vein Pattern Capturing - This module captures the dorsal vein patterns of a user with NIR camera attached with the system sharing the central database of insured persons.

Finding ROI - This determine the region of interest (in block of $320 \times 240$ ) where maximum feature points are available for better matching.

Image Enhancement - To eliminate the dark region of the input image, a morphological operation is used as

$$
\emptyset_{f}^{(s B)}=\max \{\min (f(x+b))\}
$$

where, $s B$ represents structuring element $B$ of size $s, f$ is input image and $b \in s B$.

Morphological operation is done to obtain dark vein patterns on which contrast enhancement is applied. The contrast enhancement is done using $A \times A$ window. Mathematically, the contrast enhancement (output image $G$ ) is done by

$$
\begin{aligned}
& G=255 \frac{\left[\alpha_{A\left(\phi_{f}\right)-}{ }^{\alpha_{A}\left(\phi_{f_{\text {min }}}\right)}\right]}{\left[\alpha_{A\left(\phi_{\max }\right)-}{ }^{\alpha}{ }_{A\left(\phi_{f_{\text {min }}}\right)}\right)}, \\
& \propto_{A}\left(\emptyset_{f}\right)=\left[1+\exp \left(\frac{m_{A}-\emptyset_{f}}{\sigma_{A}}\right)\right]^{-1} .
\end{aligned}
$$

$\emptyset_{f \min }$ and $\emptyset_{f \max }$ represent the minimum and maximum intensities, $m_{A}$ and $\sigma_{A}$ represent the mean and variance inside the window area.

Object Identification with Central Database - For identification of an unconscious patient, the hospital personnelscan the dorsal of left and/or right hand. The scanner is connected with central database of vein patterns of insured users from different insurance companies. NIR Camera of $850 \mathrm{~nm}$ is used for capturing dorsal vein images. For dorsal vein identification at hospital end, SIFT feature matching algorithm is used. The process of verification of unconscious patient (having no physical identity) is shown in figure 10 . 


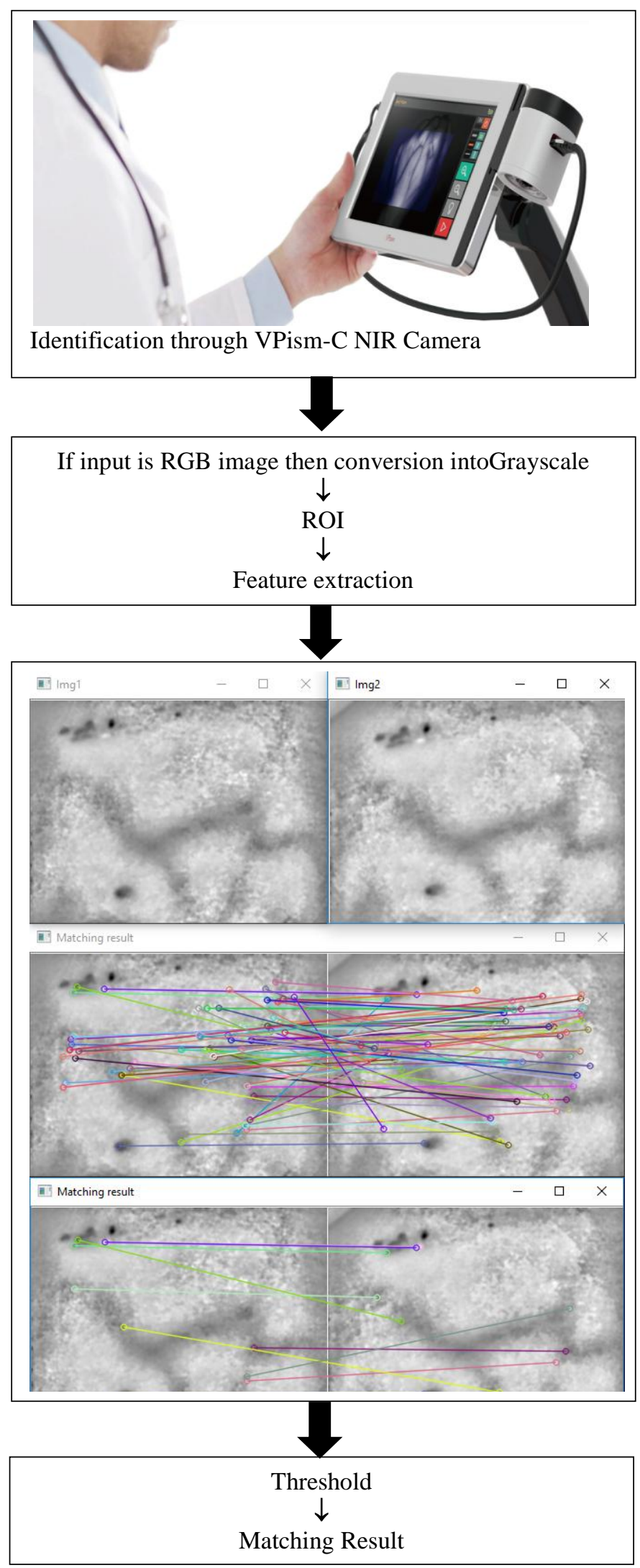

Fig. 10. Identification of patient for cashless treatment

Python 3.7.3 with OpenCv package is used as software tool. After image capturing by dedicated scanner(s), the remaining operations (pre- and post-processing) are ensured by the software tool. The pre-processing of image includes image enhancement and noise removal; while the post-processing of image includes feature extraction, matching and result analysis.

\section{RESULTS AND DisCUSSION}

For experimental purpose, the data of 250 persons was considered. Total 4 images of a person ( 2 images of left hand L1, L2) and 2 images of right hand R1, R2) were captured after an interval of 30 minutes. Total images we used for this research work were 1000 and total matching come out from 10,00000 comparisons. We had total imposters score (male, female and kids $)=980000$ results, and total genuine score (male, female and kids) $=20000$. The FAR (false accept rate) was calculated as:

$$
F A R=\frac{\text { Number of samples false accepted at a threshold }}{\text { Total number of attempts }}
$$

Table-I. FAR of full data Imposter scores

\begin{tabular}{|c|c|c|}
\hline S. No. & Threshold $\%$ & FAR \\
\hline 1 & 40 & 0.0794 \\
\hline 2 & 50 & 0.0277 \\
\hline 3 & 60 & 0.0092 \\
\hline 4 & 70 & 0.0021 \\
\hline 5 & 80 & 0.00 \\
\hline 6 & 90 & 0.00 \\
\hline
\end{tabular}

The FRR (false reject rate) was calculated as: $F R R=\frac{\text { Number of true rejected at a threshold }}{\text { Total number of attempts }}$

Table-II. Total genuine score

\begin{tabular}{|c|c|c|}
\hline S. No. & Threshold $\%$ & FRR \\
\hline 1 & 40 & 0.040 \\
\hline 2 & 50 & 0.045 \\
\hline 3 & 60 & 0.049 \\
\hline 4 & 70 & 0.080 \\
\hline 5 & 80 & 0.115 \\
\hline 6 & 90 & 0.220 \\
\hline
\end{tabular}

The overall performance of the proposed system calculated on total imposter results $=979500$ with FAR at threshold $40 \%=0.0793$. Almost similar performance was found on threshold more than $40 \%$. The overall performance $P$ is calculated as

$P=\frac{(\text { Total imposter results }-F A R)}{\text { Total results }}$

$=(979500-0.0794) \times 100 / 1000000$

$=97.95 \%$

The performance of the system at threshold 50\%, 60\%, $70 \%, 80 \%$ was found $97.99 \%$ and at threshold $90 \%$ it was found $98 \%$. Therefore, the average performance of the system was $97.99 \%$.

The plot of FAR and FRR from Table I and II is shown in figure 11 to calculate EER. The EER of the system obtained was $0.0435 \%$ which is better than results obtained in [20] [22]. 


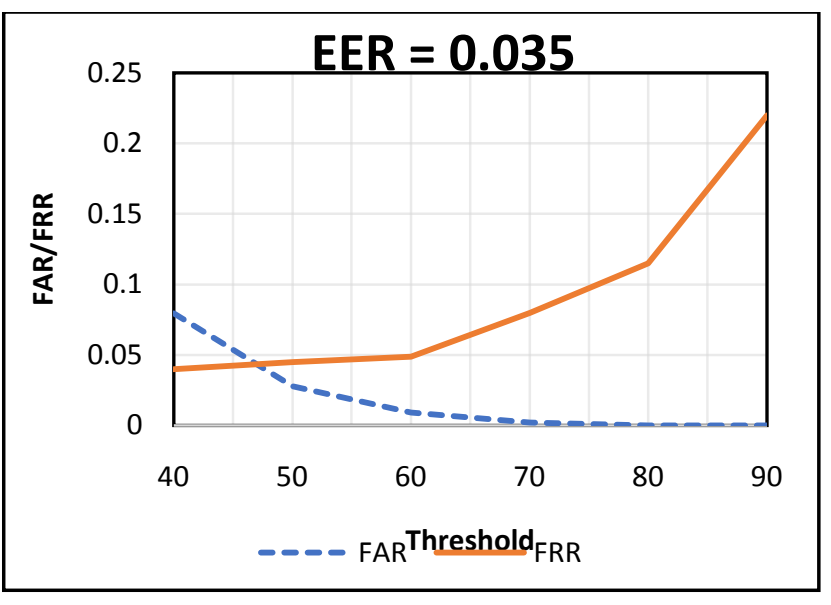

Fig. 11. Determining EER from FAR and FRR graph

For category based matching, we have divided matching into three parts:

(i) Male, female and kids matching scores with their left and right images,

(ii) Male, female and kids matching scores matched with the same group, and

(iii) Male, female and kids matching scores matched with all samples.

With reference to (i), we obtained male, female and kids matching scores with their left and right hand dorsal vein images as - total number of genuine score for male $=3200$ (for left), total number of genuine score for male $=3200$ (for right), total number of genuine score for female $=3200$ (for left), total number of genuine score for female $=3200$ (for right), Total number of genuine score for kids $=3600$ (for left), and Total number of genuine score for kids $=3600$ (for right).

Table-III Category wise FRR

\begin{tabular}{|c|c|c|c|c|c|c|}
\hline \multirow{2}{*}{ 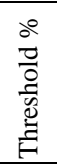 } & \multicolumn{2}{|c|}{ Male Dorsal } & \multicolumn{2}{|c|}{ Female Dorsal } & \multicolumn{2}{|c|}{ Kids Dorsal } \\
\hline & $\begin{array}{c}\text { Left } \\
(3200)\end{array}$ & $\begin{array}{l}\text { Right } \\
(3200)\end{array}$ & $\begin{array}{c}\text { Left } \\
(3200)\end{array}$ & $\begin{array}{l}\text { Right } \\
(3200)\end{array}$ & $\begin{array}{c}\text { Left } \\
(3600)\end{array}$ & $\begin{array}{l}\text { Right } \\
\text { (3600) }\end{array}$ \\
\hline 40 & 0.0000 & 0.0000 & 0.0000 & 0.0000 & 0.0000 & 0.0000 \\
\hline 50 & 0.0000 & 0.0000 & 0.0000 & 0.0000 & 0.0000 & 0.0000 \\
\hline 60 & 0.0000 & 0.0000 & 0.09375 & 0.03125 & 0.0000 & 0.0280 \\
\hline 70 & 0.0000 & 0.0000 & 0.09375 & 0.03125 & 0.0000 & 0.0280 \\
\hline 80 & 0.0000 & 0.0000 & 0.1875 & 0.1250 & 0.0280 & 0.0830 \\
\hline 90 & 0.0625 & 0.0000 & 0.2500 & 0.21875 & 0.0550 & 0.0555 \\
\hline
\end{tabular}

As (ii), we obtained Male, female and kids matching scores (excluding genuine results) matched with the same group as total number of imposter score for kids $=122400$, total number of imposter score for male $=96000$, and total number of imposter score for female $=96000$.

Table-IV Category wise FAR in same group

\begin{tabular}{|c|c|c|c|}
\hline Threshold \% & $\begin{array}{c}\text { Male Dorsal } \\
96000 \text { Samples }\end{array}$ & $\begin{array}{c}\text { Female Dorsal } \\
96000 \text { Samples }\end{array}$ & $\begin{array}{c}\text { Kids Dorsal } \\
122400 \\
\text { Samples }\end{array}$ \\
\hline 30 & 0.0114 & 0.4270 & 0.1300 \\
\hline 40 & 0.0000 & 0.0250 & 0.0370 \\
\hline 50 & 0.0000 & 0.0042 & 0.0155 \\
\hline 60 & 0.0000 & 0.0021 & 0.0024 \\
\hline 70 & 0.0000 & 0.0000 & 0.0000 \\
\hline 80 & 0.0000 & 0.0000 & 0.0000 \\
\hline 90 & 0.0000 & 0.0000 & 0.0000 \\
\hline
\end{tabular}

As (iii). We obtained Male, female and kids matching scores (excluding genuine results) matched with all samples of dorsal vein patterns as -total number of imposter score for kids $=352800$, total number of imposter score for male adults $=313600$, and total number of imposter score for female adults $=313600$.

Table-V FAR for Males, Females and Kids (all samples)

\begin{tabular}{|c|c|c|c|}
\hline $\begin{array}{c}\% \\
\text { Threshold }\end{array}$ & $\begin{array}{c}\text { Matching of } \\
\text { Male } \\
\text { (313600) to } \\
\text { all Samples }\end{array}$ & $\begin{array}{c}\text { Matching of Feale } \\
\text { (313600) to all } \\
\text { Samples }\end{array}$ & $\begin{array}{c}\text { Kids to all } \\
352800 \\
\text { Samples }\end{array}$ \\
\hline 30 & 0.055 & 0.041 & 0.091 \\
\hline 40 & 0.016 & 0.014 & 0.027 \\
\hline 50 & 0.001 & 0.002 & 0.001 \\
\hline 60 & 0.001 & 0.000 & 0.001 \\
\hline 70 & 0.000 & 0.000 & 0.000 \\
\hline 80 & 0.000 & 0.000 & 0.000 \\
\hline 90 & 0.000 & 0.000 & 0.000 \\
\hline
\end{tabular}

\section{CONCLUSION}

The accuracy of the proposed system isfound to be $97.95 \%$. The system rejected all imposter matching at $70 \%$ and above threshold as mentioned in tables IV and V. This system is capable to help in hassle free claim of medical insurance. Also, the system will identity if there is any fake claim. The results may vary due to acquisition of input image from different NIR cameras. After an interval of 05 and 10 years the vein patterns should be updated for kids and adults respectively. As this work does not include how much the vein patterns of kids may vary in 2-3 years, so as future scope this work may be done. Also, the future work needs to include database of vein patterns of persons having skin diseases and hypertension.

\section{REFERENCES}

1. Mishra Kamta Nath, Mishra Kanderp Narayan, Agarwal Anupam (2016). Vein Based Personal Identification System: A Review. International Journal of Intelligent System and Applications, 10, pp. $68-85$.

2. Montiel Ignacio Irving Morales, Aurturo J. Olvera-Lopez, Manuel Martin Ortiz, Eber E. Orozco Guillen (2014). Hand Vein Infrared Image Segmentation for Biometric Recognition. Research in Computer Science, 80, pp. 55-66.

3. Tarshi Jain, Rajendra Kumar (2019). A Study of Vein Recognition System. Acta Informatica Malaysia (AIM), 3(1), pp. 13-15.

4. Jain A K., Karthik Nand Kumar, Ross Arun (2016). 50 years of Biometric Research: Accomplishments, challenges and opportunities. Pattern Recognition Letters, Elsevier, 79, pp. 80-105.

5. Swati K. Jichkar, K. P. Kamble (2016). A Survey on Palm Vein Recognition", International Journal of Advanced Research in Electrical, Electronics and Instrumentation Engineering, 5(10), pp. 7859-7861.

6. Bango Chao Liu, Shan Juan Xie, Dong Sun Park (2016). Finger Vein Recognition using Optimal Partitioning Uniform Rotation Invariant LBP Descriptor. Hindawi Publishing Corporation-Journal of Electrical and Computer Engineering, pp. 1-10.

7. Sen Lin, Tianyang Xu, Xinyong Yin (2016). Region of Interest Extraction for Palmprint and Palm Vein Recognition. Proceedings of the IEEE International Congress on Image and Signal Processing, BioMedical Engineering and Informatics (CISP-BMEI 2016), pp. 538-542.

8. Parthsarthi De, Dibyendu Ghoshal and Tapajyoti Deb (2016). Dual Authentication of a Human Being from Simultaneous Study of Palm Patterns and IRIS Recognition. Indian Journal of Science and Technology, 9(35), pp. 1-6.

9. Jaekwon Lee, Senghwan Moon, Juhun Lim, Min-Joo Gwak, Jae Gwan Kim, Euiheon Chung and Jong-Hyun Lee (2017). Imaging of the Finger Vein and Blood Flow for Anti-Spoofing Authentication using a Laser and a MEMS Scanner. Sensors, 17(925), pp. 1-9. 
10. Gupta Jugal Kishor, Kumar Rajendra (2010). An efficient ANN Based approach for Latent Fingerprint Matching. International Journal of Computer Application, 7(10), pp.18-21.

11. Maleika Mehr Nigar M. Heenaye, R. K. Subramanian (2009). A study of Dorsal vein pattern for biometric security. University of Mauritius Research Journal, 15, 17-25.

12. R. Raghavendra, Jayachander Surbiryala, Christoph Busch, "Hand Dorsal Vein Recognition: Sensor, Algorithms and Evaluation", IEEE International Conference on Imaging Systems and Techniques (IST), 2015, pp. 1-6.

13. Khusnuliawati Hardika, Fatichah chastine, Soelaiman Rully (2017). Multi-Feature Fusion Using SIFT and LEBP for Finger Vein Recognition. Telkomnika Telecommunication, Computing, Electronics and Control, 15, pp. 478-485.

14. Wang Guoqing, Wang Jun (2017). SIFT based Vein Recognition Model: Analysis and Improvement. Computational and Mathematical Methods in Medicine, pp. 1-14.

15. Hans Verghese Mathews (2016) 'Flaws in the UIDAI Process', 51(9), available

[online]www.epw.in/journal/2016/9/special-articles/flaws-uidai-proce ss.html

16. Naidile S., Shrividya G. (2015). Personal Recognition Based on Dorsal Hand Vein Pattern. International Journal of Innovative Research in Science, Engineering and Technology, 4(5), pp. 3189-3196.

17. Ahuja Karan, Bose Abhishek, Dey Kuntal, Nagar Seema, Ferdous A Barbhuiya (2016). ISure: User Authentication in Mobile Devices using Ocular Biometrics in Visible Spectrum. IEEE International Conference on Image Processing (ICIP) Challenge Session.

18. M. Usman Akram Hassan Moatasam Awan, Abdullah Amaan Khan (2014). Dorsal Hand Veins based Person Identification. Proceedings of the $4^{\text {th }}$ International Conference on Image Processing Theory, Tools and Applications (IPTA), pp. 1-6.

19. David Mulyono, Horng Shi Jinn (2008). A Study of Finger Vein Biometrics for Personal Identification", Proceedings of International Symposium on Biometrics and Security Technologies, ISBN: 978-1-4244-2427-6.

20. Kumar, A., \& Prathyusha, K. V. (2009). Personal Authentication Using Hand Vein Triangulation and Knuckle Shape. IEEE Transactions on Image processing, 18(9), pp. 2127-2136.

21. Uriarte-Antonio, J., Hartung, D., Pascual, J. E. S., \& Sanchez-Reillo, R. (2011). Vascular Biometrics Based on a Minutiae Extraction Approach. In IEEE International Carnahan Conference on Security Technology (ICCST), pp. 1-7

22. Yuksel, A., Akarun, L., \& Sankur, B. (2011). Hand Vein Biometry Based on Geometry and Appearance Methods. IET Computer Vision, 5(6), pp. 398-406.

23. Bethanny Janney J., Sindu Divakaran, G. Umashankar (2016). Finger Vein Recognition System for Authentication of Patient Data in Hospital. International Journal of Pharma and Bio Science, 8(2), pp. 5-10

24. Pierre-Olivier Ladoux, Christophe Rosenberger and Bernadette Dorizzi (2009). Palm Vein Verification System Based on SIFT Matching. Proceedings of the $3^{\text {rd }}$ International Conference on Advances in Biometrics, Springer-Verlag Berlin Heidelberg, pp. 1290-1298.

25. Modris Greitans, Mihails Pudzs and Rihards Fuksis (2010). Palm Vein Biometrics Based on Infrared Imaging and Complex Matched Filtering, Proceedings of the $12^{\text {th }}$ ACM workshop on Multimedia and Security, pp. 101-106.

26. Ahmed Mona A., EI-Horbaty El-Sayed M., Abdel-Badeeh M. Salem (2015). Intelligent Techniques for Matching Palm Vein Images.Egyptian Computer Science Journal, 39, pp. 1-14.

27. Huafeng Qin, Lan Qin, Xue Lian, Xiping He, Chengbo Yu, Liang Xinyuan (2013). Finger-Vein Verification Based on Multi-Features Fusion.Sensors, 13(11), pp. 15048-15067

28. Jose Anand, T. G. Arul Flora, Philip Any Susan (2013). Finger-Vein Based Biometric Security.International Journal of Research in Engineering and Technology, 2(12), pp. 197-200.

\section{AUTHORS PROFILE}

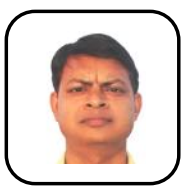

Rajendra Kumarobtained B.E. and M. Tech. in Computer Science and Engineering from Bundelkhand University, Jhansi and Uttar Pradesh Technical University, Lucknow (now Dr. APJ Abdul Kalam technical University), respectively, and currently he research-scholar School of Engineering and Technology at Sharda University, Greater Noida. He has published more 16 papers in International Journals and presented 08 papers in International Conferences held in India, Singapore, UAE, Malaysia, Thailand and Indonesia. He is author of five textbooks including Theory of Automata, Languages \& Computation from McGraw Hill Education. His area of interest includes Biometrics, Theoretical Computer Science, Software Engineering, Cyber Security, etc.

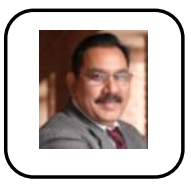

Ram Chandra Singhis Professor of Physics in School of Basic Sciences and Research, Sharda University, Greater Noida. His area of interest includes study of phase transitions in molecular liquids using density-functional theory; Time-series analysis using wavelets and talks in several conferences.

Biometrics. He has delivered keynote address and invited

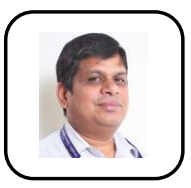

Ashok Kumar Sahoois Professor of Computer Science at Graphic Era University, Dehradun. He has 21 years of teaching and research experience.He completed M. Tech. from Uttar Pradesh Technical University, Lucknow (now Dr. APJ Abdul Kalam technical University), and Phd from Sharda University, Greater Noida. His area of interest includes Digital Image Processing, Software Engineering, Algorithms, etc. 